\title{
Comunicación
}

\section{Trasplante de microbiota fecal en un paciente con parvovirosis canina: reporte de caso clínico}

\author{
Trasplante de microbiota fecal en un paciente con parvovirosis canina: reporte de \\ caso clínico
}

\author{
Juan Carlos Pinilla León ${ }^{1,2}$, Angel Alberto Florez ${ }^{1}$
}

\section{Resumen}

El presente estudio muestra el caso de una cachorra de cuatro meses de edad que ingresó a la consulta de la clínica veterinaria «Vet Center», Bucaramanga, Colombia, por un cuadro gastroentérico severo. La paciente mostraba signos de depresión, vómito, deshidratación y fiebre. La valoración hematológica reveló una ligera anemia, y la prueba de parvovirus canino resultó positiva. Para la estabilización y tratamiento de la paciente se colocó un catéter intravenoso para el suministro de soluciones endovenosas de Ringer lactato, antibióticos, antieméticos y vitaminas. Además, se realizó como terapia adicional un trasplante de microbiota fecal al cabo de 12 horas de hospitalización, repitiéndose a las 12 horas, dando resultados satisfactorios que se discuten en este reporte clínico.

Palabras clave: canino, microbiota, parvovirus

\section{AbSTRACT}

It is reported the case of a four-month-old puppy that was admitted to the consultation at the veterinary clinic «Vet Center», Bucaramanga, Colombia, due to a severe gastroenteric disturbance. The patient showed signs of depression, vomiting,

\footnotetext{
${ }^{1}$ Facultad de Ciencias Agrícolas y Veterinarias, Universidad de Santander, Bucaramanga,Colombia

${ }^{2}$ E-mail: jcpinilla@hotmail.com
}

Recibido: 17 de febrero de 2021

Aceptado para publicación: 27 de agosto de 2021

Publicado: 27 de octubre de 2021

CLos autores. Este artículo es publicado por la Rev Inv Vet Perú de la Facultad de Medicina Veterinaria, Universidad Nacional Mayor de San Marcos. Este es un artículo de acceso abierto, distribuido bajo los términos de la licencia Creative Commons Atribución 4.0 Internacional (CC BY 4.0) [https:// creativecommons.org/licenses/by/4.0/deed.es] que permite el uso, distribución y reproducción en cualquier medio, siempre que la obra original sea debidamente citada de su fuente original 
dehydration, and fever. The haematological evaluation revealed slight anaemia, and the canine parvovirus test was positive. For the stabilization and treatment of the patient, an intravenous catheter was placed for the infusion of intravenous lactated Ringer's solutions, antibiotics, antiemetics, and vitamins. In addition, a faecal microbiota transplant was performed as additional therapy after 12 hours of hospitalization, repeating at 12 hours, giving satisfactory results that are discussed in this clinical report.

Key words: canine, microbiota, parvovirus

\section{INTRODUCCIÓN}

La Gastroenteritis Viral Canina (GVC) es una enfermedad de alta incidencia a nivel mundial con alta complejidad en la etiología, y que causa graves daños patológicos en camadas de perros (Decaro y Buonavoglia, 2012). En la actualidad, varios patógenos virales están relacionados con esta enfermedad, incluidos el parvovirus canino ( $\mathrm{PvC})$, el coronavirus canino $(\mathrm{CoV})$ y el virus del distemper canino (DC) (Decaro y Buonavoglia, 2012). El PvC pertenece al género Parvovirus, familia Parvoviridae, y causa una enfermedad muy contagiosa y mortal en los perros, especialmente cachorros (Qi et al., 2020). Los perros que se infectan muestran la enfermedad en un plazo de 3 a 7 días, y presentan gastroenteritis grave, letargo, vómitos, fiebre y diarrea, generalmente con sangre (Nelson et al., 1979; Parrish, 1995).

El parvovirus canino $(\mathrm{PvC})$ se conoce desde finales de la década del $70 \mathrm{y}$, a pesar de la vacunación intensiva, este virus todavía representa una de las principales causas de gastroenteritis hemorrágica y muerte en cachorros (Voorhees et al. 2020). La prevención de la infección por $\mathrm{PvC}$ se basa en el uso de vacunas a virus vivo modificado, que son capaces de estimular respuestas inmunes mediadas tanto por anticuerpos como por células, induciendo una protección fuerte y duradera contra la exposición posterior con cepas virulentas (Ford et al., 2017). Sin em- bargo, no siempre la vacunación contra $\mathrm{PvC}$ da como resultado el desarrollo de inmunidad activa contra este virus, lo que conduce a fallas en la inmunización que permiten que los perros vacunados estén expuestos a la infección y la enfermedad (Decaro y Buonavoglia, 2012).

Las fallas en la inmunización representan una de las principales razones de la circulación continua de CPV y pueden deberse a persistencia de la inmunidad materna en el momento de la vacunación, ausencia de respuesta inmunológica en el cachorro vacunado y a la circulación de diferentes variantes antigénicas del virus (Decaro et al., 2020). El tratamiento del $\mathrm{PvC}$ es en gran medida de apoyo y sintomático. Los componentes principales del tratamiento incluyen fluidoterapia, tratamiento con antibióticos, tratamiento antiemético y apoyo nutricional; sin embargo, otras medidas pueden incluir tratamientos antivirales y analgésicos (Mylonakis et al., 2016), y recientemente se ha propuesto el uso de trasplante de microbiota intestinal como terapia alternativa en la gastroenteritis hemorrágica por PvC (Pereira et al., 2018).

\section{Caso Chinico}

Se describe el caso clínico de un canino, hembra, raza Pastor Ovejero de 4 meses y $15 \mathrm{~kg}$ de peso corporal, que ingresó a la consulta externa de la Clínica Veterinaria «Vet Center», en Bucaramanga, Colombia, por un 
cuadro de gastroenteritis hemorrágica. El propietario manifestó que su perra presentaba heces diarreicas, vómito frecuente y falta de apetito desde hace 12 horas. De acuerdo con la anamnesis, la paciente había sido desparasitada hace tres meses con $1 / 2$ tableta de OneÒ (una tableta contiene $500 \mathrm{mg}$ de Febendazol $+50 \mathrm{mg}$ de Praziquantel +150 mg de Toltrazuril), además de haber recibido una dosis de vacuna Procyon ${ }^{\circledR}$ Dog PV MSD (vacuna a virus vivo modificado que contiene una cepa tipo $2 \mathrm{~b}$ de Parvovirus canino: 1 $\mathrm{ml})$ a los 50 días de edad.

\section{Examen Clínico}

La paciente se mostraba deprimida, deshidratación de $6 \%$, membranas mucosas ligeramente pálidas y decaimiento general. La temperatura corporal (TC) estaba en $39.3^{\circ} \mathrm{C}$, frecuencia respiratoria (FR) de 40 respiraciones/minuto y una frecuencia cardíaca (FC) de 85 latidos/minuto. El paciente tenía pulso arterial fuerte (arteria femoral) y los linfonódulos superficiales no mostraban alteraciones patológicas. Al examen clínico del sistema digestivo se apreció aumento del borborigmo intestinal confirmando la presencia de un cuadro gastroentérico.

Con base a la información obtenida en la historia médica y el examen clínico, se estableció como diagnósticos diferenciales gastroenteritis por parvovirus canino, parasitismo gastrointestinal y enteritis bacteriana. Por lo tanto, se solicitó una hematología completa, examen coprológico y una prueba rápida en placa (CPV Ag Test Kit Bionote ${ }^{\circledR}$ ) $($ sensibilidad $=100 \%$ y especificidad $=98.5 \%)$ para el diagnóstico de parvovirus canino. En vista de tratarse de un caso de urgencias, se procedió a internar el paciente en la clínica veterinaria para su manejo clínico hospitalario.

\section{Manejo del Paciente}

El paciente se hospitalizó el 15 de enero de 2021. Inicialmente se canalizó con catéter $\mathrm{N}^{\circ} 22 \mathrm{G}$ (Jelco IV Catheter Radiopaque) para la administración de fluidos y tratamiento sintomático. De acuerdo con los signos mostrados se realizó tratamiento convencional (Mylonakis et al., 2016) con suministro de Ringer lactatoÒ, en dosis de $100 \mathrm{ml}$ a una tasa de velocidad de una gota por segundo, siendo repetida cada seis horas de manera de corregir el estado de deshidratación y mantener la volemia y electrolitos en el paciente. Además, se suministró Aminolyte ${ }^{\circledR}$ (solución inyectable de vitaminas, electrolitos, minerales y dextrosa), a razón de $5 \mathrm{ml} / \mathrm{kg} /$ día. Para el control del vómito se aplicó Anemex ${ }^{\circledR}$ (solución inyectable de metoclopramida al $0.5 \%$ ) a razón de $0.5 \mathrm{mg} / \mathrm{kg}$ c/6 h $(1.5 \mathrm{ml})$ por vía endovenosa, y ranitidina (ampollas de $50 \mathrm{mg} / 2 \mathrm{ml}$ ) por vía endovenosa c/12 h a razón de $2 \mathrm{mg} / \mathrm{kg}(0.6 \mathrm{ml})$.

Para el control de infecciones secundarias bacterianas se empleó Baytril ${ }^{\circledR}$ (enrofloxacina al $5 \%$ ) a razón de $10 \mathrm{mg} / \mathrm{kg}$ $(1.5 \mathrm{ml})$ por vía endovenosa $\mathrm{c} / 24 \mathrm{~h}, \mathrm{y}$ Triseptil ${ }^{\circledR}$ (trimetroprin + sulfametazina al $24 \%)$ a razón de $10 \mathrm{mg} / \mathrm{kg}(0.6 \mathrm{ml})$ por vía endovenosa $\mathrm{c} / 24 \mathrm{~h}$. Como terapia adicional se empleó un trasplante de microbiota intestinal (Pereira et al., 2018), para lo cual se seleccionó un perro de raza Poodle completamente sano al cual se le colectó espontáneamente una muestra fecal (aproximadamente $10 \mathrm{~g}$ ) en una bolsita limpia. La muestra se mezcló y homogenizó con $10 \mathrm{ml}$ de solución salina fisiológica, y empleando una sonda de alimentación Nelaton \# 8 se introdujo totalmente la mezcla por vía rectal. El procedimiento se realizó 12 horas después de haber ingresado la paciente a hospitalización, y se repitió a las 12 horas.

\section{Resultados de Laboratorio}

Al examen coprológico directo con solución salina se observaron huevos de nematodos, además de presencia de bacterias, detritus celulares y restos de mucosa. La prueba de $\mathrm{PvC}$ resultó positiva (Figura 1). Los resultados de la hematología se muestran en el Cuadro 1. En el frotis de sangre se observaron abundantes macroplaquetas. 
Cuadro 1. Valores hematológicos en paciente canino con gastroenteritis por parvovirus canino $(\mathrm{PvC})$

\begin{tabular}{lll}
\hline Parámetro & Resultado & Valor referencial $^{1}$ \\
\hline Hemoglobina $(\mathrm{mg} / \mathrm{dl})$ & 12 & $12-18$ \\
Hematocrito $(\%)$ & 36 & $37-54$ \\
Leucocitos $\left(\mathrm{x} \mathrm{mm}^{3}\right)$ & 6,450 & $7,000-14,000$ \\
Fórmula diferencial & & \\
Neutrófilos $(\%)$ & 62 & $70-75$ \\
Linfocitos $(\%)$ & 25 & $10-20$ \\
Eosinófilos $(\%)$ & 5 & $2-4$ \\
Basófilos $(\%)$ & 3 & $0-2$ \\
Monocitos $(\%)$ & 1 & $0-2$ \\
En banda $(\%)$ & 4 & 0 \\
\hline Plaquetas $(\mathrm{x} \mathrm{mm})$ & 370,000 & $150,000-350,000$ \\
\hline
\end{tabular}

${ }^{1}$ Pedrozo et al. (2010)

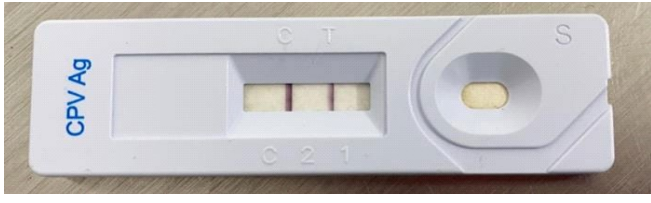

Figura 1. Resultado de la prueba para parvovirus canino - PvC (la presencia de dos bandas a color rojo «T» $\mathrm{y}$ «C» en la ventana de resultados, indica resultado positivo).

\section{Discusión}

De acuerdo con la información clínica y resultados de laboratorio obtenidos se diagnostica una gastroenteritis por parvovirus en una cachorra Pastor Ovejero de cuatro meses. A pesar del tratamiento convencional instaurado en la paciente, se recurrió al trasplante de microbiota fecal como terapia adicional. No se identificaron molestias en el paciente durante el procedimiento ni hubo necesidad de sujeción física o sedación, lo que coincide con los resultados reportados por Burton et al. (2016). En el presente caso se apreció una resolución de la gastroenteritis hemorrágica (diarrea) en menos de 24 horas del trasplante fecal. Indudablemente, la respuesta rápida fue beneficiosa para la cachorra, ya que se disminuyó el tiempo de hospitalización y tratamiento, además de la sobrevivencia del animal. Pereira et al. (2018) indican que este tratamiento reduce los costos y estancias hospitalarias con reducción del riesgo de infecciones, además de otras complicaciones y muerte de los animales.

El PvC infecta las células de las criptas de las vellosidades intestinales disminuyendo la absorción y aumentando la permeabilidad intestinal, ocasionando el cuadro gastroentérico (McCaw y Hoskins, 2006). El mecanismo de cómo el trasplante de microbiota intestinal ayuda en la resolución del cuadro entérico con diarrea mediada por el virus es incierto. Aunque la evidencia científica sobre el efecto que tiene el trasplante es escasa, la respuesta al procedimiento probablemente esté relacionada con la reconstitución de la microbiota intestinal y sus metabolitos correspondientes (Allenspach et al., 2010; Honneffer et al., 2014). 
Es indudable que la terapia de sostén empleada en el presente caso ayudó en la mejoría de la cachorra; sin embargo, sería necesario hacer estudios donde se evalúe el microbiota intestinal de cachorros, para entender el mecanismo de respuesta de la microbiota intestinal en casos de $\mathrm{PvC}$ a fin de obtener protocolos de trasplante de microbiota con un enfoque estandarizado. Las tasas de mortalidad en cachorros con infección por $\mathrm{PvC}$ pueden alcanzar el $36 \%$ en perros que reciben tratamiento estándar y hasta el $91 \%$ en animales no tratados. Por tanto, la terapia de trasplante de microbiota intestinal a partir de muestras fecales de perros sanos podría representar una alternativa, ya que muestra beneficios como ser un tratamiento efectivo, práctico, fácil y de bajo costo para veterinarios en su práctica clínica.

\section{Agradecimientos}

Los autores agradecen al propietario del paciente por la colaboración prestada en la ejecución del presente trabajo clínico. También se agradece a la Universidad de Santander por el apoyo prestado en la redacción del presente artículo.

\section{Literatura Citada}

1. Allenspach K, House A, Smith K, McNeill F, Hendricks A, ElsonRiggins J, Riddle A, et al. 2010. Evaluation of mucosal bacteria and histopathology, clinical disease activity and expression of Toll- like receptors in German shepherd dogs with chronic enteropathies. Vet Microbiol 146: 326335. doi: 10.1016/j.vetmic.2010.05.025

2. Burton EN, O'Connor E, Ericsson AC, Franklin CL. 2016. Evaluation of fecal microbiota transfer as treatment for postweaning diarrhea in research- colony puppies. JAmAssoc LabAnim 55: 582-587.
3. Decaro N, Buonavoglia C. 2012. Canine parvovirus - A review of epidemiological and diagnostic aspects, with emphasis on type 2c. Vet Microbiol 155: 1-12. doi: 10.1016/j.vetmic.2011.09.007

4. Decaro $N$, Buonavogliaa $C$, Barr $V$. 2020. Canine parvovirus vaccination and immunisation failures: are we far from disease eradication? Vet Microbiol 247: 108760. doi: 10.1016/j.vetmic.2020.1 08760

5. Ford R, Larson L, McClure K, Schultz R, Welborn L. 2017. AAHA canine vaccination guidelines. J Am Anim Hosp Assoc 53: 243-251. doi: 10.5326/ JAAHA-MS- 6741

6. Honneffer J, Minamoto Y, Suchodolski J. 2014. Microbiota alterations in acute and chronic gastrointestinal inflammation of cats and dogs. World J Gastroentero 20: 16489-16497. doi: 10.3748/wjg.v20.i44.16489

7. McCaw DL, Hoskins JD. 2006. Canine viral enteritis. In: Greene CE (ed). Infectious diseases of the dog and cat. $3^{\text {rd }}$ ed. St Louis, USA: Saunders Elsevier. p 63-70.

8. Mylonakis ME, Kalli I, Rallis TS. 2016. Canine parvoviral enteritis: an update on the clinical diagnosis, treatment, and prevention. Vet Med 7: 91-100. doi: 10.2147/VMRR.S80971

9. Nelson D, Eustis S, McAdaragh J, Stotz I. 1979. Lesions of spontaneous canine viral enteritis. Vet Pathol 16: 680686. doi: 10.1177/030098587901600606

10. Parrish CR. 1995. Pathogenesis of feline panleukopenia virus and canine parvovirus. Bailliere Clin Haem 8: 57-71. doi: 10.1016/S0950-3536(05)80232-X

11. Pedrozo R, Quintana GI, Bazán A, Florentín M. 2010. Valores hematológicos de referencia en caninos adultos aparentemente sanos, que concurren a una clínica privada de Asunción. Mem Inst Investig Cienc Salud 8: 5-13. 
12. Pereira G, Gomes L, Santos L, Alfieri A, Weese J, Costa M. 2018. Fecal microbiota transplantation in puppies with canine parvovirus infection. J Vet Intern Med 32: 707-711 doi: 10.1111/jvim. 15072

13. Voorhees I, Lee H, Allison A, LopezAstacio R, Goodman L, Oyesola $O$, Omobowale O, et al. 2020. Limited intrahost diversity and background evolution accompany 40 years of canine parvovirus host adaptation and spread. J Virol 94: e01162-19. doi: 10.1128/ JVI.01162-19

14. Qi S, Zhao J, Guo D, Sun D. 2020. A mini-review on the epidemiology of canine parvovirus in China. Front Vet Sci 7: 5. doi: 10.3389/fvets. 2020.00005 